\title{
A geometric proof of Stallings' theorem on groups with more than one end
}

Graham A. Niblo (g.a.niblo@maths.soton.ac.uk) Faculty of Mathematical Studies, University of Southampton, Highfield, Southampton SO17 1BJ, UK

\begin{abstract}
Stallings showed that a finitely generated group which has more than one end splits as an amalgamated free product or an HNN extension over a finite subgroup. Dunwoody gave a new geometric proof of the theorem for the class of almost finitely presented groups. Here we adapt the method to the class of finitely generated groups using Sageev's generalisation of Bass Serre theory concerning group pairs with more than one end.
\end{abstract}

Keywords: amalgamated free product, HNN extension, singularity obstruction, CAT(0) cube complex, Bass Serre theory, Ends, Stallings' theorem

AMS Classification: Primary 20E34; Secondary 20F32, 05C25

Dedicated to John Stallings on the occasion of his 66.6th birthday.

\section{DRAFT VERSION}

\section{Introduction}

A group $G$ is said to split over a subgroup $K$ if it admits a non-trivial decomposition as an amalgamated free product $G=A *_{K} B$ or an HNN extension $G=A *_{K}$. In [18], [19] Stallings showed that a finitely generated group $G$ splits over a finite subgroup if and only if $G$ has more than one end.

Stallings remarked in [18] that he was led to the proof by consideration of Papakyriokopoulos's sphere theorem for 3-manifolds which may be understood in terms of minimal surface theory.

In [5] Dunwoody gave a geometric proof of Stallings' theorem for the class of (almost) finitely presented groups, based on a combinatorial analogue of the least area surface technique. The principle advantage of combinatorial least area surfaces (or their analogues, least weight tracks in a 2-complex) is that their existence is trivially guaranteed without appeal to Meeks - Yau minimal surface theory. On the other hand, Meeks - Yau theory does guarantee (via the Meeks - Yau rounding trick) that minimal surfaces cannot intersect transversly which is not true for combinatorial minimal surfaces. Nonetheless Dunwoody developed a 
"cut and paste" technique for these objects which bypassed the difficulty and allows one to prove the equivariant sphere and loop theorems in the category of PL 3-manifolds as well as Stallings' theorem. [3].

In the following statement of Dunwoody's result $H_{c}^{1}\left(X, \mathbb{Z}_{2}\right)$ denotes cohomology with compact support and coefficients in $\mathbb{Z} / 2 \mathbb{Z}$. This cohomology group measures the number of topological ends of the space $X$.

THEOREM (Dunwoody) If $G$ is a group acting properly discontinuously on a locally finite 2-complex $X$ with $H^{1}\left(X, \mathbb{Z}_{2}\right)=0$ and $H_{c}^{1}\left(X, \mathbb{Z}_{2}\right) \neq$ 0 then $X$ contains a least weight track $\tau$ such that the orbit $G \tau$ consists of a disjoint family of translates of $\tau$.

To obtain Stallings' theorem one applies this theorem to the presentation complex (the Cayley 2-complex) of the group. Since this complex is simply connected $H^{1}\left(X, \mathbb{Z}_{2}\right)=0$ and the hypothesis that $G$ has more than one end translates into the fact that $H_{c}^{1}\left(X, \mathbb{Z}_{2}\right) \neq 0$. There is a tree dual to the pattern of tracks in the $G$-orbit of $\tau$ on which the group acts with unbounded orbits and the edge stabilisers are the stabilisers of the tracks. Since the tracks are finite and $G$ acts properly these stabilisers are finite and applying Bass-Serre theory one obtains the required splitting of the $G$ over a finite subgroup.

In order to generalise Dunwoody's method to the class of finitely generated groups one needs to construct an appropriate replacement for the Cayley complex since it is not, in general, locally finite. With a little more work Dunwoody's method can be made to apply with the less restrictive condition that the 2-complex should be locally finite away from its 0-skeleton, but even this will not be enough to allow us to use the Cayley complex in the proof.

The key idea in this paper is that we may use the 2-skeleton of the Sageev complex, a contractible cubical complex which may be associated to the group $G$. [14].

THEOREM Let $G$ be a finitely generated group with $e(G) \geq 2$. Then there is a $C A T(0)$ cubical complex $X$ on which $G$ acts, such that $G$ has one orbit of hyperplanes, each hyperplane is compact, hyperplane stabilisers are finite, and $G$ has an unbounded orbit.

A cubical complex is a cell complex in which each cell is identified with a Euclidean cube of the appropriate dimension, and the glueing maps are taken to be isometries. Such a complex admits a geodesic metric and it is this metric which satisifes the CAT(0) inequality in the statement of the theorem above.

In a cubical complex each cube contains a family of codimension-1 subspaces passing through its centre called midplanes; these restrict 
to midplanes in each face. Passing from cube to adjacent cube the midplanes match up to form immersed codimension-1 subspaces known as hyperplanes. One can show that in a CAT(0) cubical complex each hyperplane is a (totally geodesic) separating codimension-1 subspace [11].

Although the 2-skeleton of the Sageev complex has almost all the properties we need to apply Dunwoody's technique directly there is one technical difficulty introduced by the fact that the complex is generically not locally finite. In particular one can no longer detect ends of the complex using cohomology with compact support. However by analysing the hyperplane structure of the complex one can show that it is locally finite away from its 0-skeleton, and we will show how to adapt Dunwoody's technique to deal with complexes satisfying this less restrictive condition.

The technique described in this paper can also be applied to obtain a new proof of the following generalisation of Stallings' theorem which was known to Dunwoody and Roller [6]; it plays a key role in the proof of the algebraic torus theorem [7]. The result was (re)proved in [10] by appealing to Dunwoody's result on cuts in graphs [4]. To state the generalisation we will need the following definitions from [15].

Let $G$ be a finitely generated group and $H$ a subgroup of $G$. Given a finite generating set for $G$ define the number of ends of the pair $H<G$ to be the number of topological ends of the quotient of the corresponding Cayley graph of $G$ by the subgroup $H$. Scott showed in [15] that this number, which we will denote by $e(G, H)$, is independant of the generating set chosen, and that for $G$ to split over $H$ it is necessary for $e(G, H)$ to be at least 2 . Unfortunately it is possible for $e(G, H)$ to be at least 2 without $G$ splitting over $H$. There is an obstruction to the splitting, whose definition depends on a choice (of $H$-almost invariant set, see [15]), which is supported on a union of double cosets $H F H$, where $F$ is a finite subset if $H$ is finitely generated. We can now state the generalisation, which is the main theorem in this paper:

THEOREM A Let $G$ be a finitely generated group with a finitely generated subgroup $H$ such that $e(G, H) \geq 2$ and $H$ has non-empty singularity obstruction $H F H$ for some finite subset $F$ in $G$. If the subgroup $\langle H F H\rangle$ lies in the commensurator of $H$ in $G$ then $G$ splits over a subgroup commensurable with $H$.

In order to prove this generalisation of Stallings' theorem we will use the following result from [10]:

FINITE HYPERPLANE THEOREM Let $G$ be a finitely generated group and $H<G$ be a finitely generated subgroup with $e(G, H) \geq 2$. If 
the singularity obstruction HFH (for some choice of $H$-almost invariant set) lies inside the commensurator $\left\{g \in G \mid H^{g}\right.$ is commensurable with $\left.H\right\}$ then $G$ acts on a $C A T(0)$ cubical complex $X$, with the following properties:

1. There is one orbit of hyperplanes $G \mathcal{H}$,

2. The hyperplanes are compact

\section{3. $H$ is a hyperplane stabiliser}

4. There is an unbounded vertex orbit.

Theorem A follows directly from the finite hyperplane theorem together with Theorem B:

THEOREM B Let $G$ be a finitely generated group which acts on a $C A T(0)$ cubical complex $X$, such that $G$ has one orbit of hyperplanes, each hyperplane is compact, and $G$ has an unbounded orbit. Then $G$ splits over a subgroup commensurable with a hyperplane stabiliser.

In section 2 of this paper we begin the proof of Theorem B. We will define the notion of a least weight track in a triangulated 2-complex, and show that the 2-skeleton of the cube complex under consideration contains least weight tracks. We also prove that if two least weight tracks intersect then we can carry out a canonical surgery on the two tracks to obtain a pair of non-intersecting least weight tracks. There are two natural ways to complete the proof, Dunwoody's original method, adapted to our context, is given in section 4 , where we show how to carry out the surgery on an entire orbit of least weight tracks to obtain a $G$-equivariant family of disjoint least weight tracks (a so-called $G$ pattern). There is a natural tree, known as the dual tree associated to this pattern and the action of $G$ on this tree will give rise to the required splitting of the group. An alternative more geometric method, is based on ideas of Jaco, Rubinstein and Casson, and that version is given in section 3 . In this approach the notion of a least weight track is strengthened to that of a minimal track, and one can show that minimal tracks cannot cross. Once existence of minimal tracks is established it follows that the orbit of a minimal track is a $G$-pattern giving rise to the required splitting. The reader therefore has a choice. Those with a taste for geometry will find a complete proof in sections 2 and 3, and those with a preference for combinatorial methods may prefer to read sections 2 and 4 . Finally in section 5 we sketch an example given by the action of $P G L_{2}(\mathbb{Z})$ on a $\mathrm{CAT}(0)$ cube complex associated to its natural action on the hyperbolic plane. We will show how the natural 
decomposition of $P G L_{2}(\mathbb{Z})$ as an amalgamated free product may be read from the geometry of the cube complex.

\section{Tracks in 2-complexes}

In this section we will begin the proof of:

THEOREM B Let $G$ be a finitely generated group which acts on a $C A T(0)$ cubical complex $X$, such that $G$ has one orbit of hyperplanes, each hyperplane is compact, hyperplane stabilisers are finite, and $G$ has an unbounded orbit. Then $G$ splits over a subgroup commensurable with a hyperplane stabiliser.

In outline we attack the problem by studying the action of $G$ on the 2-skeleton $X$ of the cube complex $Z$. Since $Z$ is contractible $H^{1}\left(X, \mathbb{Z}_{2}\right)=$ $H^{1}\left(Z, \mathbb{Z}_{2}\right)=0$.

We begin by choosing a $G$-equivariant triangulation of the 2-complex $X$. For technical reasons we would like to choose the triangulation so that it refines the cubical structure and so that no vertices lie in a hyperplane. Unfortunately we may not be able to do this if cell stabilisers act non-trivially, since we may be forced to use the centre of a cube as a vertex. To get round the problem we first barycentrically subdivide the cube complex which has the effect of duplicating all the hyperplanes as parallel pairs. Now cell stabilisers act trivially and we can triangulate in the required manner. Finally we note that the single orbit of hyperplanes may have been replaced by two orbits, but in this case every edge of the complex is within a bounded distance of each orbit and this is sufficient to carry out the proof.

Equip $X$ with the piecewise Euclidean path metric obtained by identifying each 2-simplex with an equilateral Euclidean triangle. A subset of $X$ will be said to be unbounded if it is not contained in any ball of finite diameter in this metric.

A pattern in the 2-complex consists of a closed subset $\mathcal{P}$ in the complement of the 0 -skeleton of $X$ such that $\mathcal{P}$ meets each closed 1-simplex $\gamma$ in a finite union of points all lying in the interior of $\gamma$, and each closed 2 -simplex in a finite union of disjoint closed line segments. Note that this definition does not quite agree with that given by Dunwoody in [5], since we allow the line segments to join two points in the same edge of a 2 -simplex.

A track is a connected pattern. A pattern (or track) is said to be finite if it intersects only finitely many 1 -simplices, and in this case we assign it a weight given by the number of points in the intersection with the 1-skeleton. The pattern is said to be bounded if it is contained in a ball of finite diameter in $X$. 
A track is said to be essential if it is finite and its complement has two unbounded components. A pattern is said to be essential if at least one of its component tracks is essential, and it is said to be a least weight pattern if it is essential and has least weight among all essential patterns. Note that a least weight pattern must be a track, since otherwise each of its component tracks must have strictly smaller weight, and at least one of these must be essential contradicting the minimality of the weight of the original pattern. By varying the argument given in [9], (using cohomology with bounded support in place of cohomology with compact support) we will obtain the following observation.

LEMMA 1. Any least weight pattern on $X$ consists of a single track which intersects each edge of $X$ in at most one point and each 2-simplex in at most a single arc joining distinct edges of the simplex.

Proof. Given a pattern $\mathcal{P}$ we can define its characteristic function, which is a function $z$ from $X^{(1)}$ to the positive integers, counting the number of points on each 1-simplex lying in $\mathcal{P}$. This function defines $\mathcal{P}$ up to reasonable equivalence, and, as Dunwoody observed, if we reduce its values mod 2 , we obtain a 1-cocycle; one can show that the corresponding class in $H^{1}\left(X, \mathbb{Z}_{2}\right)$ is zero if and only if the pattern separates $X,[2$, section VI, 3$]$. If $\mathcal{P}$ is finite, in that it meets only finitely many edges, then

Let $\left\{\tau_{1}, \ldots, \tau_{n}\right\}$ be the components of $\mathcal{P}$, with characteristic functions $z_{1}, \ldots, z_{n}$. If these are all zero as classes in $H_{b}^{1}\left(X, \mathbb{Z}_{2}\right)$ then so is $[z]$ contradicting the definition of $\mathcal{P}$, hence at least one of the tracks $\tau_{i}$ is itself a non-trivial pattern. Its weight is at least $\|\mathcal{P}\|$ since $\mathcal{P}$ is minimal, and at most $\|\mathcal{P}\|$ since $\tau_{i} \cap X^{(1)}$ is a subset of $\mathcal{P} \cap X^{(1)}$. Hence $\tau_{i}=\mathcal{P}$.

It follows that $\mathcal{P}$ is a single track which separates $X$ into two unbounded components.

It is a consequence of [2, section VI, theorem 3.2] that any function $z$ from $X^{(1)}$ to the positive integers, such that the reduction of $z \bmod 2$ is a cocycle (call it $w$ ), gives rise to a pattern on $X$ whose characteristic function is $w$. Reducing the characteristic function of $\mathcal{P} \bmod 2$ we obtain a new function with values 0 and 1 , from which we can build a new pattern $\mathcal{Q}$. If $\mathcal{P}$ crossed some edge at least twice then the norm of $\mathcal{Q}$ is strictly less than $\|\mathcal{P}\|$, but their characteristic functions give identical elements of $H_{b}^{1}\left(X, \mathbb{Z}_{2}\right)$ by construction, so $\mathcal{Q}$ is also a minimal pattern, contradicting the minimality of $\mathcal{P}$.

Of course we don't yet know that $X$ admits any essential tracks, but we may show that it does as follows. Each hyperplane intersects the triangulation in a track and since the hyperplane is compact each 
of these tracks is finite. Since the hyperplane separates $Z$ into two unbounded components, this track does the same to $X$, so it is essential as required.

Since $X$ is simply connected and contains an essential pattern we can choose a minimal pattern in $X$, which as noted above consists of a single track $\tau$. Two such minimal patterns $\sigma$ and $\tau$ may of course intersect, but by perturbing them an arbitrarily small amount we can ensure that all the intersections occur in the interior of 2-cells, and this perturbation may be chosen so that it does not alter the weights of the two tracks. We will show how to carry out cut and paste operations on the tracks at these intersection points to obtain two new minimal tracks which do not intersect.

THEOREM 2. Let $\sigma$ and $\tau$ be minimal tracks in a simply connected 2-complex $X$ which intersect transversely in the interior of the 2-cells of $X$. Then there are disjoint minimal tracks $\sigma^{\prime}$ and $\tau^{\prime}$ in $X$ such that $(\sigma \cup \tau) \cap X^{(1)}=\left(\sigma^{\prime} \cup \tau^{\prime}\right) \cap X^{(1)}$.

Proof. If $\sigma$ and $\tau$ are disjoint there is nothing to prove so assume that they are not disjoint. Since $\sigma$ and $\tau$ are bounded and each separates $X$ into two unbounded components the same is true for small regular neighbourhoods $N(\sigma)$ of $\sigma$ and $N(\tau)$ of $\tau$. We may choose these neighbourhoods so that their union $N$ is a (compact) regular neighbourhood of the connected 1-complex $\sigma \cup \tau$ which avoids the 0 -skeleton of $X$. Since $N$ is compact its boundary is a finite pattern $\mathcal{P}$ in $X$ which intersects the 1-skeleton of $X$ in two points for each point in the intersection $(\sigma \cup \tau) \cap X^{(1)}$. The weight of $\mathcal{P}$ is therefore $2(w(\sigma)+w(\tau))$.

Denote the two (unbounded) components of $X-N(\sigma)$ by $S^{+}$and $S^{-}$, and the two (unbounded) components of $X-N(\tau)$ by $T^{+}$and $T^{-}$. Any two points in a component of the complement of $N$ can be joined by a path in $X$ which crosses neither $\sigma$ nor $\tau$, so any such component is contained entirely in one of the subsets $S^{+} \cap T^{+}, S^{+} \cap T^{-}, S^{-} \cap T^{+}$, or $S^{-} \cap T^{-}$. Since $\mathcal{P}$ is finite its complement has only finitely many components and it follows that each of the four subsets $S^{ \pm} \cap T^{ \pm}$is a union of finitely many components of $X-\mathcal{P}$.

If the two subsets $S^{+} \cap T^{+}$and $S^{-} \cap T^{-}$are both bounded then both subsets $S^{+} \cap T^{-}, S^{-} \cap T^{+}$must be unbounded to ensure that both $S^{+}$and $S^{-}$are unbounded. By switching $S^{+}$and $S^{-}$and $T^{+}$and $T^{-}$if necessary we can assume that both $S^{+} \cap T^{+}$and $S^{-} \cap T^{-}$are unbounded. It follows that at least one component of $X-\mathcal{P}$ (which we will denote $C^{+}$) is unbounded and lies inside $S^{+} \cap T^{+}$and another (which we will denote $C^{-}$) is unbounded and lies inside $S^{-} \cap T^{-}$. The boundary of $C^{+}$in $X$ is a component $\sigma^{\prime}$ of $\mathcal{P}$ which separates the unbounded set $C^{+}$from its complement, which contains the unbounded 


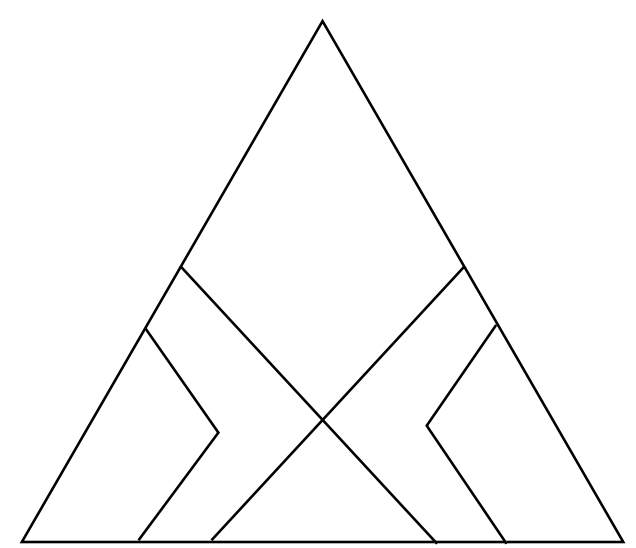

Figure 1. The canonical cut and paste replaces crossing arcs with a disjoint pair.

set $C^{-}$. Hence $\sigma^{\prime}$ is an essential pattern and must have weight at least $n$. Similarly the boundary of $C^{-}$is also an essential pattern of weight at least $n$, and we will denote it by $\tau^{\prime}$.

Given an edge $e$ of $X$ which intersects $N$, the subset $N \cap e$ cuts $e$ into two or three regions depending on whether $e$ intersects one or both of the tracks $\tau$ and $\sigma$. Notice first that adjacent regions of an edge cannot both be in $C^{+} \cup C^{-}$since they are separated by only one of the tracks. Furthermore if $e$ intersects just one of the tracks $\sigma, \tau$ then at most one of the regions can lie in the subset $C^{+} \cup C^{-}$, since the two subsets are separated by both tracks. Hence the edge also intersects at most one of the two tracks $\sigma^{\prime}, \tau^{\prime}$, and does so in a single point. If $e$ intersects both $\sigma$ and $\tau$ then either the central region of the edge lies in $C^{+} \cup C^{-}$, or the two outside ones each lie in one of them. In either case the two patterns $\sigma^{\prime}, \tau^{\prime}$ intersect the edge in exactly two points between them. It follows that the total weight of the two patterns $\sigma^{\prime}$ and $\tau^{\prime}$ is at most $w(\sigma)+w(\tau)=2 n$. Since each has weight at least $n$ they both have weight exactly $n$ and are therefore minimal tracks. It follows from the lemma that neither of them intersects a 2-simplex in an arc joining an edge to itself. Furthermore if $\sigma \cup \tau$ intersects a given edge in $k$ points $(k=1$ or 2$)$ then so does $\sigma^{\prime} \cup \tau^{\prime}$ for otherwise $2 n=w\left(\sigma^{\prime}\right)+w\left(\tau^{\prime}\right)<w(\sigma)+w(\tau)=2 n$.

Now define a cut and paste operation as follows. If the tracks $\sigma, \tau$ cross in a 2-simplex so that they both intersect the edge $e$ then replace the arcs of $\sigma, \tau$ in the 2-simplex by the two arcs in the boundary of the regular neighbourhood $N$ which separate the end points of $e$ and join it to the two other edges of the 2-simplex. The operation is illustrated in Figure 1. 
The argument above shows that carrying out this cut and paste operation at every intersection must yield precisely the two tracks which we have chosen and no other components. Finally we observe that the pattern $\sigma^{\prime} \cup \tau^{\prime}$ may be isotoped to have the same intersection with the 1-skeleton of $X$ as the pattern $\sigma \cup \tau$. We will call it the canonical cut and paste operation.

\section{The geometric method}

In this section we will make use of a method initially developed by Jaco and Rubinstein [13] and Casson which strengthens the notion of a least weight track to that of a minimal track with the objective of ensuring that minimal tracks which intersect are coincident. It will follow that the $G$ orbit of a minimal track is a $G$-equivariant pattern and the $G$ action on the dual tree to this pattern will induce a splitting of $G$ over the stabiliser of the minimal track.

In order to define minimal tracks we will need the notion of an ideal 2-complex.

An ideal 2-simplex is a topological 2-simplex with its vertices removed, which is identified with an ideal hyperbolic triangle in the Poincaré disc, with vertices at the points $e^{(2 \pi / 3)}, e^{(4 \pi / 3)}$ and 1 on the circle at infinity. Each edge of the ideal 2-simplex has a midpoint which is the point on that edge closest to the centre of Poincaré's disc under the identification.

Given a 2-dimensional simplicial complex $X$ we obtain an ideal 2complex $Y$ by removing the vertices and uniformizing the remains of each 2-simplex as an ideal 2-simplex. We adjust the glueing maps as necessary to ensure that they are hyperbolic isometries matching the midpoints of the corresponding edges; uniformising the 2-cells in this way we may impose a path metric on $Y$ and the matching condition ensures that the metric is complete. Since tracks do not intersect the 0 -skeleton of $X$ any finite track in $X$ gives rise to a finite track $\tau$ in $Y$ which we can isotop, relative to the intersection of $\tau$ with the edges of $Y$, to a track in which the intersection with any cell is a union of hyperbolic geodesics. We assign a length to any finite track $\tau$ in $Y$ by summing the length of all the geodesic arcs thus obtained. We define the complexity of the track $\tau$ to be the ordered pair $(w, \ell)$ where $w$ is the total number of points in $\tau \cap Y^{(1)}$ and $\ell$ is the length of $\tau$. If a track lies entirely in the link of a vertex we may isotop it so that its intersection with any 2-cell is (under the uniformization) the arc of a horocycle. Such a track will be called a horocyclic track, and has the 
property that its length can be arbitrarily shortened by pushing it off towards the vertex at infinity in whose link it lies.

We define the complexity of a track $\tau$ to be the ordered pair $(w, \ell)$ where $w$ is the weight of the track as measured in $X$ and $\ell$ is its length in $Y$. We order tracks lexicographically by complexity. A track in $Y$ is ssaid to be minimal if it is a least weight track in $X$ and achieves the minimum complexity. Our 2-complex $X$ contains a least weight track $\tau$, and we would like to show that $Y$ contains a minimal track.Unfortunately as we have already remarked if a least weight track lies in the link of a vertex then it may be isotoped to least weight tracks of arbitrarily small length, so the infimum of complexities will not be achieved. Nonetheless we will show that it is possible to obtain a splitting of the group even in this case, and that provided there are no horocyclic essential tracks in $Y$ then $Y$ will contain a minimal track. The proof of theorem B then depends on a variant of the Meeks-Yau rounding trick which ensures that tracks of minimal complexity cannot cross. In fact the two cases break down as follows, either $Y$ contains a least weight track of length $\ell<\log (1+\sqrt{2})$ or every least weight track has length at least $\log (1+\sqrt{2})$.

Case $1 \ell<\log (1+\sqrt{2})$. Any track which does not lie in the link of a vertex in $X$ must cross a pair of adjacent triangles joining disjoint edges. We can embed the union of the two triangles in the hyperbolic plane so as to preserve the length of paths in the union. It follows that the arc in $\tau$ connecting the two edges must have (hyperbolic) length greater than $\log (1+\sqrt{2})$ (the distance between the two geodesics in the hyperbolic plane), which contradicts the fact that $\ell<\log (1+\sqrt{2})$.

Since $\tau$ has length less than $\log (1+\sqrt{2})$ it must lie in the link of some vertex $\mathrm{v}$ and we may isotop the track so that it consists of short horocyclic arcs centered at $v$ in the cells it crosses. Since these horocyclic arcs are determined uniquely by any point they contain, so is the resulting track. It follows that translates of this horocyclic track which intersect actually co-incide, so $G$ acts equivariantly on the orbit of the horocyclic track to produce a $G$-pattern.

Case 2 Now suppose that any least weight track in $Y$ has length at least $\log (1+\sqrt{2})$, and let $\ell$ be the infimum of lengths of essential tracks of weight $w$. Choose a horocyclic neighbourhood $N_{0}$ of the ideal vertices of $Y$ such that the distance from any point within the neighbourhood to a point on the opposite side of the triangle is at least $2 \ell$. Then choose a smaller horocyclic neighbourhood $N_{1}$ so that the distance from the original horocycle to any point in this 
sub-neighbourhood is also at least $\ell$. It follows that for sufficiently small $\varepsilon$ any essential track of complexity at most $(w, \ell+\varepsilon)$ which enters the neighbourhood $N_{1}$ must remain in the neighbourhood $N_{0}$. If it does so then it may be isotoped to a horocyclic track and its length shortened arbitrarily, which contradicts our assumption that essential tracks have weight at least $\log (1+\sqrt{2})$. Hence if we remove the open sub-neighbourhood $N_{1}$ of the ideal vertices we obtain a closed subcomplex which contains all the essential tracks of complexity at most $(w, \ell+\varepsilon)$.

This sub-complex is locally finite, since all infinite branching in $X$ occurs at the vertices, which have been removed, and if we choose an edge $e$ and take the intersection of the subcomplex with the ball of radius $\ell+\varepsilon$ around $e$ we obtain a compact subset $C$ of $Y$ which contains all non-horocyclic essential tracks of complexity at most $(w, \ell+\varepsilon)$ intersecting the edge $e$.

Now any (geodesic) minimal track contained in $C$ may be regarded as an equicontinuous map of a finite graph, with $|w|$ vertices, into $C$. There are, up to homeomorphism, only finitely many such graphs, so given any infinite sequence of minimal tracks we can choose an infinite subsequence such that the corresponding graphs are all homeomorphic.

Choose a sequence of minimal tracks with weight $w$ and length converging to $\ell$. Choose an infinite subsequence for which the underlying graphs are all homeomorphic to some fixed graph $\Gamma$ and the tracks all have length at most $\ell+\varepsilon$. We can apply the the Arzela- Ascoli theorem to the corresponding sequence of maps $\tau_{i}: \Gamma \longrightarrow C$. Without loss of generality we can assume that each arc of the track $\tau_{i}(\Gamma)$ is geodesic.

Arzela- Ascoli Theorem[1] If $C$ is a compact metric space and $\Gamma$ is a separable metric space, then every sequence of equicontinuous maps $f_{n}: \Gamma \longrightarrow X$ has a subsequence that converges uniformly on compact subsets to a continuous map $f: \Gamma \longrightarrow C$.

Each edge of the graph $\Gamma$ is mapped to a geodesic crossing a single ideal 2-cell at each stage in the sequence, and since the geodesics are bounded away from the (ideal) vertices by the constraint that the tracks lie in the subcomplex $C$, in the limit edges of the graph are still mapped to geodesics of non-zero length. Hence the limit yields a pattern of weight $w$ contained in $C$, and since length varies continuously the limit is a minimal track of length $\ell$. Hence minimal tracks exist in $Y$. 
To complete the argument it remains to show that minimal tracks never cross one another. This is achieved by a PL version of the Meeks-Yau rounding trick used by Jaco and Rubinstein [8]. The details of the method, used in a context similar to the one we need, can be found in Lemma 2.7 of [16]. We give a brief outline here. Assume that two minimal tracks $\sigma$ and $\tau$ do have non-empty intersection. We may isotop the two tracks by an arbitrairly small amount to ensure that the intersection is transverse and lies away from the 1-skeleton, by moving the vertices of the tracks slightly. This will increase the total length, but not the weight, of the two tracks, but, because length deforms continuously, we can ensure that the total length change is arbitrarily small. Indeed, to a first order approximation it is zero as a function of the distance moved by the vertices of the track. Now that the intersection has been moved off the 1-skeleton we may cut and paste the two tracks as in section 2 to obtain two new least weight tracks. Smoothing the corners introduced by the cut and paste results in a comparatively large reduction in the total length of the two new tracks. Again, regarded as a function of the displacement of the vertices, we can estimate it to first order and the reduction is linear in the displacement with a non-zero first order term. It follows that by choosing a small enough perturbation of the tracks, cutting, pasting and rounding, we obtain two new least weight tracks with lengths whose sum is shorter than that of the orginal tracks, and so at least one of the two new tracks must be shorter than the original minimal tracks. This is a contradiction. Hence minimal tracks never intersect and it follows that the orbit of a minimal track is a $G$-invariant pattern.

We have shown that either the complex $Y$ contains a horocyclic track, or it contains a minimal track and in either case the orbit $G \tau$ of the track consists of a disjoint family of tracks, i.e., we obtain a $G$-equivariant pattern in $Y$.

Associated to this pattern there is a tree (the dual tree) defined as follows. The vertices of the tree are the components of the complement of the pattern in $X$. Two vertice are joined by an edge if the corresponding regions are adjacent in $X$, and since tracks are separating in $X$ every edge in the graph this defines is also separating. Hence we have defined a tree, and it is clear that $G$ acts on the tree with edge stabilisers the stabilisers of the tracks in the pattern.

To see that the action of $G$ on the tree has an unbounded orbit, we argue as follows. The track $\tau$ in $Y$ yields a finite track in 
$X$ which separates $X$ into two unbounded subsets. The complex also contains the hyperplane track $\sigma$ which is also bounded, and since every point of the complex is within a bounded distance of some hyperplane in a given orbit each point of $X$ is within a globally bounded distance $\delta$ of some translate of this track. Both unbounded components of the complement of $\tau$ contain edges arbitrarily far from $\tau$ and therefore contain translates $g_{i}(\sigma)$ of $\sigma$ arbitrarily far from $\tau$. But then there are translates of $\tau$ within $\delta$ of $g_{i}(\sigma)$, and by choosing the elements $g_{i}$ to ensure that $g_{i}(\sigma)$ is far enough from $\tau$ we can ensure that these translates of $\tau$ are disjoint from it. Hence the edge corresponding to $\tau$ cuts the tree into two components both of which contain translates of $\tau$ under teh action of $G$. A standard argument shows that $G$ has no global fixed point and so, according to the Bass-Serre theorem [17] the action of $G$ on the tree induces a splitting of the group over any of the track stabilisers. To complete the proof we need to show that the stabiliser of any track is commensurable with the stabiliser of a hyperplane.

If the track does not intersect any 2-cells then it consists of a single point in an edge adjacent to no 2-cells. In this case the hyperplane in $Y$ crossing that edge is just the midpoint of the edge, and coincides with the track. Since $G$ acts transitively on hyperplanes we see that the cube complex is itself a tree. In this case the group splits directly over the hyperplane stabiliser.

Otherwise note that since the track is finite its stabiliser has a finite index subgroup $H$ which fixes each cell which it crosses. Since the triangulation refines the squaring of the 2-skeleton of $Y$, if the track intersects any 2-cell then $H$ fixes a square in the original cube complex, and therefore preserves the hyperplanes crossing that square. It follows that $H$ is a subgroup of a hyperplane stabiliser. On the other hand, the stabiliser of one of these hyperplanes has a finite index subgroup which fixes the finite union of 2-cells containing minimal tracks meeting the cells it crosses. It follows that the hyperplane stabiliser $H$ has a finite index subgroup which fixes the minimal track. Hence the track stabiliser and the given hyperplane stabiliser are commensurable as required.

\section{Dunwoody's pattern summing method}

In this section we give an alternative method to complete the proof of theorem B. As in the proof of Dunwoody's Theorem 4.1 [5] we will 
construct a $G$-invariant pattern in $X$ consisting of least weight tracks by taking the pattern sum of the tracks $g \tau$ ranging over the elements $g \in G$. The proof of Dunwoody's Theorem 4.1 requires that there are only finitely many translates of $\tau$ meeting any given edge of $X$, and Dunwoody's original argument contains the hidden assumption that the 2-complex is locally finite. Fortunately the argument can be made to work provided only that the complex is locally finite away from the 0-skeleton of $X$, which gives the full strength of Dunwoody's Theorem 4.1 , and allows us to use it to complete the proof of Stallings' theorem in that style.

Our approach is to use the local finiteness properties of $X$ to show that there is a finite set of cells in $X$ such that any least weight track which intersects the given edge e is contained in the union of these cells. Consider, as in section 2, the graph $\Gamma$ dual to the triangulation of $X$ : it has vertex set $V$ consisting of the union of the 1 and 2-cells in $X$, and there is an edge joining an edge $e^{\prime}$ of $X$ to a triangle $\sigma$ in $X$ if and only if $e^{\prime}$ is a face of $\sigma$. As noted before the graph $\Gamma$ is locally finite, so for any positive integer $d$ the ball of radius $d$ around a vertex $v$ of $\Gamma$ is finite. Now any track $\tau^{\prime}$ in $X$ defines a connected subgraph of $\Gamma$, consisting of those vertices corresponding to the cells of $X$ which have non-empty intersection with $\tau^{\prime}$. If $\tau^{\prime}$ is a finite track then the subgraph is finite, and if $\tau^{\prime}$ has weight $\mathrm{d}$ and intersects the edge $e^{\prime}$, then the subgraph is contained in the ball of radius $d$ around $e^{\prime}$ in $\Gamma$. It follows that the ball of radius $m$ around an edge $e$ contains every edge and 2-cell of $X$ which intersects a least weight track crossing $e$, and that there are only finitely many such cells. Let $K_{0}$ denote the union of these cells, so that $K_{0}$ is a finite subcomplex of $X$ which contains every least weight track intersecting $e^{\prime}$. A least weight track meets any edge at most once, and is defined by the edges it does meet, so it follows that a least weight track is uniquely defined by the subgraph in $\Gamma$ which corresponds to it. It follows that there are only finitely many least weight tracks in $X$ crossing a given edge $e^{\prime}$.

Now let $\tau$ be a least weight track contained in the subcomplex $K_{0}$. Any least weight track $\tau^{\prime}$ crossing $\tau$ must share at least one edge with it, and its image in $\Gamma$ therefore intersects the image of $\tau$. It follows that the image of $\tau^{\prime}$ is contained in the ball of radius $m$ around the image of $K_{0}$, which is again a finite subgraph. This gives a finite subcomplex $K_{1}$ in $X$ which contains every least weight track $\tau^{\prime}$ crossing a least weight track in $K_{0}$. Now consider the pattern sum of the orbit of a least weight track $\tau$ under the action of $G$. The sum may be taken in two stages. First we take the pattern sum of all the tracks $g \tau$ contained in $K_{1}$. By Dunwoody's argument in [5] Theorem 3.3 this is a pattern consisting of (finitely many disjoint) least weight tracks. 
Now we take the tracks in this new pattern together with the family $\left\{g \tau \mid g \tau\right.$ is not contained in $\left.K_{1}\right\}$. The intersection of this family of tracks with the 1-skeleton of $X$ is the same as that of the original family, so the pattern sum of the new family is the same as the pattern sum of the original. But now given any of the tracks in the family which are contained in $K_{0}$, the only tracks in the family which can cross it are contained in $K_{1}$, and these are all nested by the construction in the previous step. Hence no track in the family intersects any track contained in $K_{0}$, therefore the tracks contained in $K_{0}$ are already nested and do not change in the next stage of the pattern sum. It follows that the tracks of the pattern sum contained in $K_{0}$ are least weight tracks. Since this argument applies everywhere in $X$ all the tracks in the pattern sum are least weight tracks.

Repeating the argument given at the end of section 3 we see that the track stabilisers are again commensurate with the hyperplane stabilisers in the cube complex $Z$ as required.

\section{The example of $P G L_{2}(\mathbb{Z})$}

In this section we will examine the action of the Coxeter group $P G L_{2}(\mathbb{Z})$ on a $\mathrm{CAT}(0)$ cube complex defined in [12]. Figure 2 illustrates (part of) the complex and is intended to hint at the relationship between the hyperplanes in the cube complex and the mirrors for the natural action of the group as a group generated by hyperbolic reflections. The precise relationship is that hyperplanes in the cube complex are in bijective correspondence with the mirrors in the hyperbolic plane, and that hyperplanes cross if and only if the corresponding mirrors cross. The 3-cubes correspond to the triple intersections of mirrors and the squares which are not face of 3-cubes correspond to double points. The action of $P G L_{2}(\mathbb{Z})$ on the cube complex is properly discontinuous, since the action on the plane is proper, and since the cube complex has more than one end its 2-skeleton contains essential finite tracks. Examples are given by the intersection of a hyperplane with the 2-skeleton, and in figure 3 we illustrate one of weight 8 . It should be clear from the diagram that this track crosses translates of itself under the action of the group. For example the rotation of order 3 generated by the product of two reflections in mirrors at angle $2 \pi / 3$ rotates a 3 -cube around a diagonal and carries the hyperplane to cross itself. However these hyperplanes do not yield least weight tracks. It should be clear that if we take any square of the complex which is not the face of a 3 -cube then we obtain two hyperplanes crossing it, one yielding the track of weight 8 we have already described, and the other yielding a 
track of weight 2. Since there are no isolated edges in the cubing, there are no tracks of weight 1 , so such a track must have least weight. It should be clear from inspection that the translates of this track form a $G$-pattern and yield a splitting from the action of $G$ on the dual tree. Unfortunately the action inverts edges of the dual tree (the reflection in the corresponding mirror acts as an involution switching sides of the hyperplane) so the vertex stabilisers of the splitting are not quite given by stabilisers of vertices in the tree. To extract the classical Bass-Serre splitting of the group one can adjust the cube complex in the following way.

Collapse any square of the cube complex which is not the face of a 3 -cube by collapsing in a direction parallel to its free edges. The effect is to obtain a cube complex which is still CAT(0) and which is a union of 3 -cubes. Each surviving hyperplane contracts to a union of two squares. The resulting cube complex is the Sageev complex associated to finite dihedral group $D_{3}$ stabilising the 3 -cube. This is also the stabiliser of the mirror line associated to one of the reflections.

Each least weight track in the new cube complex is a quadrilateral parallel to the edge in the intersection of two 3-cubes. This is illustrated in the right hand picture of Figure 3. If we choose the track sufficiently close to the edge then its translates under the action of the group are disjoint, as illustrated, and we obtain a $G$-equivariant pattern. The dual tree gives a splitting of the group over the stabiliser of the quadrilateral, which has index 2 in the stabiliser of the common edge to which it is parallel. But this is the same as the stabiliser of the square which contracted onto it, which is, in turn the elliptic subgroup of the Coxeter group generated by the 2 reflections in the mirrors corresponding to the two hyperplanes crossing the square. In fact the dual tree is the standard Bass-Serre tree for the decomposition of $P G L_{2}(\mathbb{Z})$ as an amalgamated free product:

$$
P G L_{2}(\mathbb{Z})=D_{2} \underset{\mathbb{Z}_{2}}{*} D_{3}
$$

The dihedral groups $D_{2}$ and $D_{3}$ in the splitting are the two finite special subgroups $\left\langle s_{1}, s_{3}\right\rangle$ and $\left\langle s_{1}, s_{2}\right\rangle$ respectively, in the standard Coxeter presentation of the group $P G L_{2}(\mathbb{Z})=\left\langle s_{1}, s_{2}, s_{3}\right| s_{1}^{2}=s_{2}^{2}=s_{3}^{2}=$ $\left.\left(s_{1} s_{3}\right)^{2}=\left(s_{1} s_{2}\right)^{3}=1\right\rangle$.

It is left to the reader to verify that the 2-skeleton of the cube complex does not admit any essential tracks of weight less than 4 and to show that the minimal track is indeed given by the quadrilateral described above. 

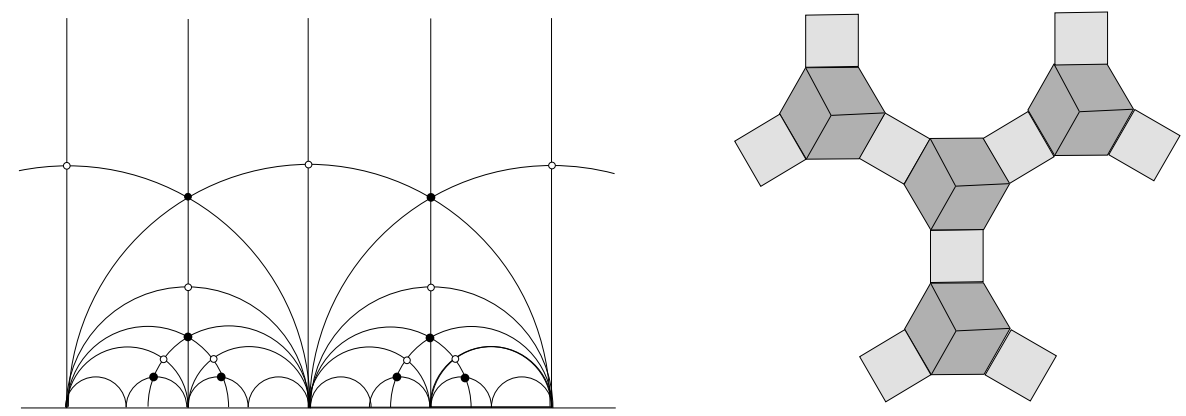

Figure 2. The Coxeter complex for the group $\mathrm{PGL}_{2}(\mathbb{Z})$ embedded in the hyperbolic plane, and the corresponding $\mathrm{CAT}(0)$ cube complex.
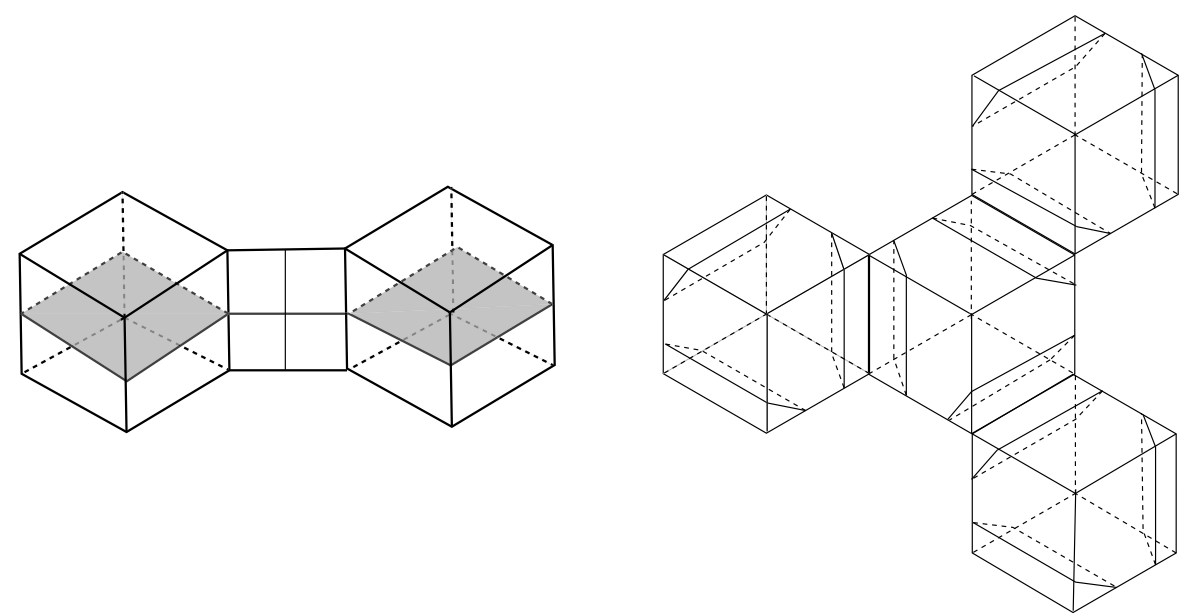

Figure 3. The hyperplane tracks of weight 2 and 8 in the cube complex and the pattern of minimal tracks in the contracted cube complex.

\section{References}

1. M. R. Bridson and A. Haefliger, Metric spaces of non-positive curvature, Springer-Verlag, Berlin(1999).

2. B.H. Bowditch, 'Groups acting on Cantor sets and the end structure of graphs', Preprint, 2000.

3. W. Dicks and M. J. Dunwoody, 'Groups acting on graphs', Cambridge University Press, 1989.

4. M. J. Dunwoody, 'Cutting up graphs' Combinatorica Vol. 2, No. 1, 1982 pp. $15-23$.

5. M. J. Dunwoody, 'The accessibility of finitely presented groups' Invent. Math. Vol. 81,1985 pp. $449-457$.

6. M. J. Dunwoody and M. A. Roller, 'Splittings of groups over polycyclic by finite groups' Bull. London Math. Soc. Vol. 25, 1993 pp.39-96.

7. M. J. Dunwoody and E. L. Swenson, 'The algebraic torus theorem', Invent. Math. 140 (2000), no. 3, pp. 605-637. 
8. W. Jaco and Hyam J. Rubinstein, 'PL-minimal surfaces in 3-manifolds', J. Differential Geom., 27, (1988)pp. 493-524.

9. G. A. Niblo, 'Finding splittings of groups and 3-manifolds', Bull. London Math. Soc. Vol. 27, 1995 pp. 567-574.

10. G. A. Niblo, 'The singularity obstruction for group splittings', to appear in Topology and its applications, Topology Appl. 119 (2002), no. 1, pp. 17-31.

11. G. A. Niblo and L. D. Reeves, 'The geometry of cube complexes and the complexity of their fundamental groups', Topology 37 (1998), no. 3, pp. 621-633.

12. G. A. Niblo and L. D. Reeves, 'Coxeter groups act on CAT(0) cube complexes', preprint, Southampton University, 2001 (12 pages).

13. W. Jaco and J. H. Rubinstein, 'PL minimal surfaces in 3-manifolds' . J. Differential Geom. 27 (1988), no. 3, pp. 493-524.

14. M. Sageev 'Ends of group pairs and non-positively curved cube complexes', Proc. London Maths. Soc.(3) Vol. 71, 1995 pp 585-617.

15. G. P. Scott, 'Ends of pairs of groups', Journal of Pure and Applied Algebra Vol. 11, 1977, pp 179-198.

16. G. P. Scott and G. A. Swarup, 'An algebraic annulus theorem', Pacific J. Math. 196, (2000), no. 2, pp. 461-506.

17. J. P. Serre, Trees, Translated from the French by John Stillwell, SpringerVerlag, Berlin (1980).

18. J. R. Stallings, 'On torsion-free groups with infinitely many ends', Ann. of Math. 88, (1968), pp. 312-334.

19. J. R. Stallings, Group Theory and 3-dimensional manifolds, Yale Mathematical Monographs, Vol. 4, Yale University Press, New Haven, (1971). 